\title{
3 Towards an embodied view of the mathematics curriculum in a world of technology
}

\author{
Thomas E. Kieren \\ University of Alberta \\ Edmonton, Canada
}

\begin{abstract}
Over the past 30 years, two views of curriculum in which ideas and techniques from informatics are used in mathematics have dominated. On one view the computer or multi-media device carries the curriculum to an individual and is programmed to evaluate the individual learning. On the second view the computer is a tool for personal construction of mathematics. This essay adds to the second view and contends that a curriculum in action must be observed as embodied, not only in the thinking of the individual, but in the interactions in the computer or multi-media environment and in the opportunities for mathematical practice it offers.
\end{abstract}

\section{Keywords}

Cognition, curriculum development, graphics/programmable calculators, knowledge representation, philosophy.

\section{INTRODUCTION: WHERE IS THE CURRICULUM?}

Thirty years ago the use of the computer was touted as having the potential to greatly affect the mathematics curriculum. One can finally today observe evidence in practical classroom terms-in the numbers and sophistication of graphing calculators in high school classrooms; in interesting multi-media research projects; on shelves of computer stores and the Internet-of the impact of computers and multi-media interactive systems on mathematical activity in schools and elsewhere. The question for this paper is "what is the nature of the 
impact of such software/hardware and courseware systems on the mathematics curriculum?"

Before turning to a discussion of the computer/informatics/mathematics curriculum, consider the following imagined examples of mathematics in action, each of which considers an aspect of fractals, a mathematical topic which owes its public popularity if not its existence to today's very high speed computers and multi-media systems.

Fractalmania A student is seated at a powerful contemporary computer with a large colour monitor. She is using a (fictitious) piece of software called 'Fractalmania'. The student is fascinated as she generates a Mandelbrot set using a menu which also allows her to generate such sets at different levels of complexity. Using the 'zoom inspector' feature she is excited and calls a friend over to see the beautiful multi-colour repeating pattern she sees as she 'zooms in'. The teacher, using a large monitor, is able to point to key elements of this fractal, generated for the whole class, such as the self-similarity across levels. The teacher encourages the students to try other examples and read about fractals in the available books in class.

Logo Fractals The students, working in pairs at a computer, are trying to get 'the turtle' to move in the path of a von Koch fractal curve. In their work and discussions they notice that this curve is made up of segments and the nature of the path is determined by recursively inter-related levels. With some assistance pairs of students create and then execute a procedure like the following:

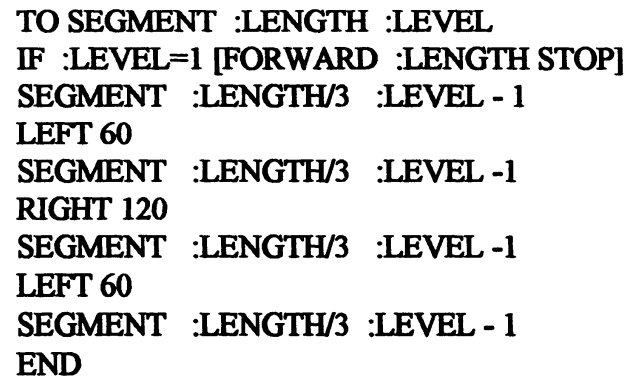

(Weston (1985), 44)

This activity leads in three other directions. One involves the study of properties of fractals (e.g., dimensionality and recursion); the second involves developing new geometric figures using SEGMENT as a sub procedure, and the third involves the definitional design of new fractal figures.

What are the differences in the mathematical activities in these two settings? In both cases students produced patterns of potential mathematical significance. But inherent in the differences in the kinds of thinking in which students engaged and in the kinds of interaction which take place are differences in what the 
computer scientist Mike Fellows (Bell et al., 1996) would call the 'mathematics vitamins' of the two settings.

The differences in the settings allow us to elaborate our previous key question as follows: "Where is the curriculum?" "What is its nature?"

As might be seen in the differences in these two settings, it is the contention of this essay that an informatics curriculum is not found solely in the hardware or courseware, nor is it solely in the creation of each student. The curriculum is a co-emergent phenomenon arising out of the interaction between students and hardware and courseware, and between students and students and teachers in such an environment. Such a view of curriculum is an embodied one.

\section{WHERE WAS THE CURRICULUM?}

In 1978 when I (Kieren, 1978) wrote about informatics and mathematics, the computer and related software were either seen as something an individual user reacted to or acted upon. Under the former view students' mathematical cognitions were viewed as answering questions or solving problems by making an accurate representation of a computer based mathematics in their heads and providing responses which could in some way be matched to pre-given standards. The computer might also be used to evaluate such a match. This view was and continues to be seen in computerised testing programs or in computational practice programs, regardless of the fact that the visual or audio feedbacks and rewards are spectacular or the data bases generated for the student, the teacher or the testing agency are elaborate. Such a representationist view of cognition also underlays most original computer assisted instruction or computer testing programs. This view of human thinking as modelled in computational terms underlies numerous contemporary 'intelligent' computer assisted instruction efforts. Under such views the curriculum is seen as developed in the programming of the computer and exists in the software itself. Mathematical knowing is in response to a pre-given, or at least pre-programmed curriculum.

Another view of informatics and mathematics which has a long history is that of the computer as a tool for learning mathematics (my focus in the 1978 paper). Such a view pre-supposed a more constructivist or constructionist view of mathematical knowing. In such cases students were typically asked to devise programs (for example in some version of Fortran or BASIC or later LOGO) to accomplish some mathematical task or to solve a problem. The computer procedure allowed students to represent their own constructed thinking in a form which allowed them to see if such thinking was viable-did their procedures work? The computer, at least metaphorically, because not all systems could actually do all of the things that teachers and their students might have liked, could be seen as providing a space for the study of algorithms or a way of a students constructing mathematics for themselves by constructing algorithms. This was certainly at the heart of the work of Arthur Engel (1976). This 
constructivist "tool" view would underlie the Fractal Logo example above and has been elaborated over the last twenty years by Papert (1980) and others such as Hoyles and Noss, (1992). Papert suggested that using the computer, and other computer controlled devices (e.g., Lego-Logo) in body syntonic and ego-syntonic ways allowed even young children access to experience with big contemporary mathematical ideas such as subprocedure, recursion and debugging. Thus this constructivist view would observe the curriculum in the child's action in a space. Rather than the computer as curriculum provider of mathematics for the child, the computer is a means by which the child builds mathematically rich experiences and reflects upon them. Thus the curriculum, rather than being seen as residing in the software, is now seen as emergent in the actions of individual students using the computer as a medium.

\section{COMPUTERS AND CURRICULUM: A SPACE FOR BRINGING FORTH A WORLD OF SIGNIFICANCE WITH OTHERS}

Under either the representationist or constructivist views discussed above it is usual to think of using the computer in teaching mathematics in task specific terms. This has been and still is particularly true when the computer is used as a tutor or tester or a drill provider even in a game setting. The user receives a prompt from the computer, responds and receives direct feedback. Interaction with the computer is pictured as a series of discreet tasks and responses.

The constructivist or constructionist views outlined above certainly challenge the view of cognition as simply responding to a singular task. But even problem solving using a utility like Cabri (Laborde et al., 1995) is task based. In using multi-media mathematics adventure programs such as Phoenix Quest from EGEMS (1996) students engage in mathematical activity which goes beyond a task orientation. Certainly some contemporary views on cognition and computing encourage a broader view as well (e.g., Winograd and Flores, 1987)).

In thinking about an informatics curriculum in an embodied way, it is useful to think of cognition not simply in task terms but as bringing forth a world of mathematical significance with others (Maturana and Varela, 1987). In particular, such 'bringing forth' and the informatics curriculum surrounding it will be seen in terms of three distinct but necessarily overlapping embodiments: that defined by the lived experience and related capabilities a person brings to a setting (in person embodiment); that which arises in interactions in a setting (person in embodiment); and that in which the person's mathematical activity is observed in mathematical cultural terms (embodiment in the body of mathematics).

In observing the in person embodiment we look for the schemes and thinking patterns which a person uses in a computer mathematics setting. For example, are there illustrations of algorithmic thinking? Does the way in which the students make and use algorithms change? Do they use algorithms or other 
computer models as representations of images that they hold? How formal and general are such representations and the debugging actions on them? This embodiment prompts us to think of human computer interaction as a space for image making, reasoning and formalising. It also prompts us to ask how the informatics curriculum becomes an occasion for such mathematical actions.

This last remark reminds us of the importance of the context in mathematical thinking. Rather than thinking of students' computer related mathematical activities in internal or individual terms, it is more useful to think of them as coemergent. Even though a person acts constructively to constitute a computer related world, that world and the features selected from it by the person are fully implicated in the mathematical actions of that person. Imagine for a moment students engaging in a mathematical adventure on the computer. They might be asked to create their own characters who have identifiable features with many possible instances of such features. Further imagine that their designed characters move through a maze of logical pathways with progress determined by logical decisions based on the features of the creatures. As we observe the human adventurer we could ask "where is the mathematical cognition?" It is clear that the constructed reasoning of the individual is fully implicated and determines the nature of the game being played. But by the same token the player is likely reasoning and using the tools of the game in ways she or he may never have done without them, i.e., person in embodiment.

This is even more the case when the person is adventuring with another person. The ways in which one person even references the mathematical actions of the other shows that the actions of the second person are implicated in the actions of the first person. The power of this second embodiment increases if two or more persons can be observed as recursively providing occasions for one another's mathematical actions. Thus in this example the curriculum is not simply the designed mathematical adventure game. Nor is it simply the path of actions of a person playing the game (or the 'sum' of the actions of all players). An enactive view of an informatic mathematics curriculum suggests that the interaction systematically enlarges the sphere of that curriculum.

The work of Fellows (Bell et al., 1996) and his colleagues suggest that the mathematical adventuring above (or any mathematical activity) can be interrogated for its 'math vitamins'. More formally this idea reflects the third embodiment, embodiment in the body of mathematics. The notion of embodiment in the body of mathematics challenges one to observe informatics/mathematics curriculums and to identify the kind and level of mathematical practices which occur in them.

As one considers mathematics curriculum which are based on informatic approaches, the discussion above suggests that one needs to look beyond the features of the software or hardware or courseware. If one believes that curriculum reveals itself in action or in the running (currare), then the three embodiments for students' informatic actions also provide a frame for observing, describing and analysing the curriculum. 


\section{THERE IS THE CURRICULUM: A TRIPLY EMBODIED INTERPRETATION}

As suggested at the outset of this essay, a teacher today is faced with a vast array of informatic approaches to, or multimedia settings for, the mathematics learning. It is beyond the scope of this essay to analyse even elements, such as spreadsheet/graphing curriculums or geometric utilities, of such a curricular collection. Instead only one such curricular setting involving the widely used graphing calculator tool will be interpreted using the triple embodiment concepts of an enactive view of mathematical activity.

\section{It depends}

Imagine the following scene ${ }^{1}$ :

A group of 15-year-old students in an average class of high school students in a suburban Canadian school are embarking on the study of systems of equations. As an early activity, the class mainly working in pairs, but each having a graphing calculator with 'trace' and 'zoom' functions, are working with the following variable entry prompt ${ }^{2}$ :

Burgers-on-the-Run has a small shop that sells burgers on a drive-up basis. The owner finds that the weekly fixed charges on the building, facilities and the drive-up is $\$ 935$. The costs in making a burger average $\$ 2.85$. The average selling price for a burger is $\$ 4.95$. Does Burgers-on-the-Run make money?

Very quickly, nearly all of the students create the functions

$$
y_{1}=2.85 x+935 \text { and } y_{2}=4.95 x
$$

which describe the cost and income properties of the situation. Figure 1 below illustrates some of the graphing activities engaged in by students. Remarks $A-G$ provide a trace of elements of the curriculum in action found in this environment.

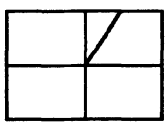

1a)

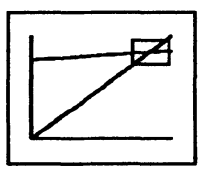

1c)

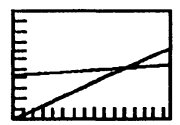

1b)

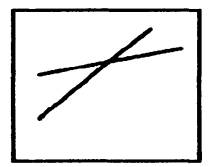

1d)

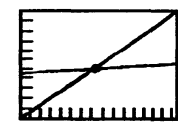

1c)

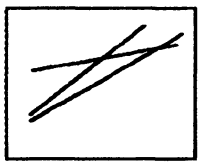

1e)

Figure 1 Some graphing calculator images. 
A. Allwyn, working alone and looking at la calls the teacher over claiming that there is no solution to the problem. Even with prompting from the teacher he cannot imagine that there is a second functional graph hidden off screen.

B. On the other hand, Kristy and John can be observed to quickly move through graphs $1 \mathrm{la}, \mathrm{lb}$ and $1 \mathrm{c}$. They realise that the domain of the function which can be seen on the screen is controllable. Even before they arrive at a 'solution' using a trace utility, the teacher has them sketch their graphs and list their 'range' selections on the board.

C. Like Allwyn, Colleen and Beth were 'stuck' at Figure la. Unlike Allwyn, when they observe Kristy and John's work, they look back at the $y_{2}=2.85 x+935$ and notice that the $y$ intercept is the problem. They too, with some fumbling trials, generate a graph like $1 \mathrm{~b}$, and attempt to use a trace function to find out the circumstances under which Burgers-on-the-Run makes money.

D. Meanwhile Kristy and John create a zoom window to take a second look at this situation (1d, e).

E. Colleen and Beth consult with them and find out how to alter their graph using a zoom box and are interested in how this helps them in their attempt to talk about whether Burger King is or is not making money (le).

At this point (when most of the students have discussed and written something about Burgers-on-the-Run making money) the teacher raises another question: "What happens when there is a sale of a dollar off on the burgers?"

F. Almost immediately both Kristy and John and Colleen and Beth type in $y_{3}=$ $3.85 \mathrm{x}$. And both pairs guess that it will take more sales to get Burgers-on-theRun into a money making position (for the sale burger). Kristy and John function in their zoom environment without visible axes making inferences for the new function based on the portion of the domain found in the zoom box (1f).

G. Colleen and Beth are puzzled by the meaning of the new graph (1f). They clear this graph and recreate a new graph using first the standard domain and range (-10 to 10$)$ and then a more tailored domain and range and to understand this 'new' situation.

This trace allows us to consider an informatics curriculum in action. Of course there are obvious elements in this curriculum - the prompt itself; the capabilities and limitations of the graphing calculator and even the follow-up prompt. But the triple embodiment perspective allows us to examine the curriculum in action differently. The first perspective is that this curriculum was different for Allwyn; Beth and Colleen; and Kristy and John. Although Allwyn (see Remark A above), like the others created appropriate input functions, his image of linear systems inhibited him from proceeding with the curriculum. He apparently thought that linear functions were the results of numerical inputs and not mathematical objects 
in their own right. If no graph appeared on the screen, there was no function. Although the environment provided potentials for action, they were not selected by him. Thus Allwyn's structure or in person embodiment helps define the nature of the curriculum, and in this case to limit it.

In Remark $\mathbf{C}$ above we can glimpse the role that the actions of others and interactions can play in an informatic curriculum. In particular, we see Colleen and Beth prompted by Kristy and John's drawings, reconsider and expand their own applications of linear functions in this setting. While like Allwyn, Colleen and Beth were influenced initially by the 'picture' on the screen, observations of Kristy and John's images and actions triggered a change in behaviour for them.

At Remark E we again see how the actions of Kristy and John become part of the curriculum for Colleen and Beth. Thus in this particular informatic situation, interaction with others (or our person in embodiment) influences the curriculum and is indeed part of it. Further, the particulars of small screen and private graphing calculator use, rather than restricting the curriculum, prompted the teacher to see the need to extend the curriculum and the nature of student-student interaction within it.

Finally we can ask in what ways the computer environment affected the embodiment in the body of mathematics for these students. We first turn to the prompt itself. Through it the teacher was implying that linear systems could be approached through the use of functions. We see this kind of 'function reasoning' developed especially in the actions of Kristy and John. The computer environment allowed four of these students to explore a particular problem engaging in functional reasoning using features of the electronic environment (e.g., axes, domain-range, approximation). But using the graphing calculator suggests some limitations on the embodiment in the body of mathematics. This functional/graphing calculator approach restricts the nature of the reasoning in this environment and particularly the linear algebraic reasoning. Thus, the computer environment both promotes and limits the kinds of mathematics that students might enact and hence impacts the curriculum.

It is usual to think of the variable entry prompt, the features of the graphing utility and perhaps the teacher's questions as constituting the curriculum in the situation above. This vignette asks us to think otherwise. The curriculum-inaction is also affected by the thinking schemes brought to it by the students. Thus Allwyn, Beth and Colleen and Kristy and John experienced different curriculums. Further, especially as seen in Colleen and Beth's actions, the curriculum occurs in the interactions and is fully implicated by them. Further interactions with the utility itself affect the curriculum. For example, although the features of the zoom box inhibited Beth and Colleen from working on mathematics abstractly, by the same token they were able to construct less abstract graphic representations of the mathematics involved. Finally even approaching the mathematics of linear systems through a graphing utility affected the nature of the mathematics experienced by the students. Thus the curriculum-in-action goes beyond the usual 
definition and includes consideration of student thinking, interactions in the environment, and embodiments in the body of mathematics, all-at-once.

\section{FINAL NOTE}

Over the past 30 to 40 years, the impact of computers on the mathematics curriculum has been viewed from two perspectives. The computer and other multimedia devices were observed as the medium which delivered the curriculum to the students in ever more elaborate and spectacular ways. A second perspective was that such devices were tools which would allow the student to construct or build her or his own mathematical ideas in enhanced ways. In particular it was thought that various computer software/hardware combinations would trigger more elaborate and more varied student mathematics.

It has been the purpose of the essay to add to these two perspectives and to challenge the first one altogether. We have to interrogate both the computer and related courseware and students in action with it (and the teachers, too) all at once to understand the curriculum. The informatics curriculum is co-emergent and fully affected by the triply embodied actions and interactions of the students enacting it. The curriculum is not in the hardware or software or courseware. It is not simply in the heads of the students or the teacher. The informatics mathematics curriculum, like 'all knowing', is in the interactions themselves.

\section{Notes}

1 This account is based on research done as part of a project supported in part by the social Sciences and Humanities Research Council of Canada grant 410-960405 .

2 The idea of variable entry prompt has been developed by Elaine Simmt at the University of Alberta to highlight two things about what otherwise might be seen as a problem solving task. First the word 'prompt' indicates that it is the student who determines and generates problems by acting selectively in the face of the prompt and the mental and environmental tools at the student's disposal and in interaction with others. 'Variable entry' implies that students at various levels of sophistication can find ways into action appropriate to the prompt. 


\section{REFERENCES}

Bell, T., Witten, I. and Fellows, M. (1996). Computer Science Unplugged-off line activities and games for all ages. http://www.eosc.canterbury.ae.nz/ tim

E-GEMS (1996). Phoenix Quest. Vancouver: Super Tangrams, E-GEMS and the University of Vancouver.

Engel, A. (1976, August). The Computer and Mathematics. Paper delivered at The Third International Congress on Mathematics Education, Karlsruhe, Germany.

Kieran, T. E. (1978). Influence of Informatics on Mathematics Teaching. In D. Johnson and D Tinsley (eds.) Informatics and Mathematics in Secondary Schools, Impacts and Relationships, 77-83.

Hoyles, C. and Noss, R. (1992). Learning Mathematics and Logo, Cambridge: MIT Press.

Laborde, C. and Laborde, J. M. (1995). What about a Learning Environment where Euclidean concepts are manipulated with a mouse? In DeSessa, Hoyles, Noss and Edwards (eds.) The Computer and Exploratory Learning. NATO ASI Series. Berlin: Springer Verlag.

Maturana, H and Varela, F. (1987). Tree of Knowledge. Boston: New Science Library.

Papert, S. (1980). Mindstorms. New York: Basic Books.

Weston, D (1985). The Second Logo Book. Glenview IL: Scott Foresman Company.

Winograd, T and Flores, F. (1987). Understanding computers and cognition. Norwood NJ: Ablex Publishing Corporation.

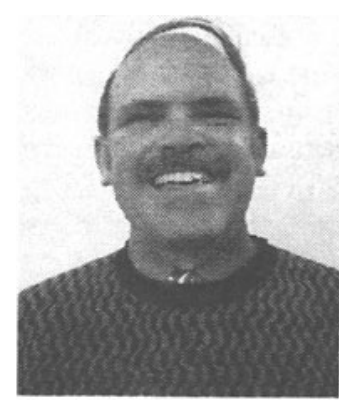

Tom Kieren is a professor of mathematics education at the University of Alberta and has had a long standing interest in informatics and mathematics. In the 1960's he wrote his doctoral thesis on using computers in Grade 11 mathematics. He offered one of the first courses on informatics and mathematics in Canada in 1968 and continued both curricular and research interests in this area over the years. He has done research or supervised research on uses of Logo in mathematics as well as on multi-media approaches to the study of recursive phenomena. 